\title{
ROUND TRIP DELAY TIME AS A LINEAR FUNCTION OF DISTANCE BETWEEN THE SENSOR NODES IN WIRELESS SENSOR NETWORK
}

\author{
Ravindra N Duche ${ }^{1}$, N.P.Sarwade ${ }^{2}$ \\ ${ }^{1}$ Research Scholar, Department of Electrical Engineering, VJTI, Mumbai, India \\ ${ }^{2}$ Department of Electrical Engineering, VJTI, Mumbai, India
}

\begin{abstract}
In a dense wireless sensor networks with portable sensor nodes identification of sensor node location, distance of particular sensor node from some reference node and fault detection are the great challenging issues. Round trip delay (RTD) time measurement technique is an easy way to obtain the information regarding above issues in WSN. But round trip delay measurement is affected by various parameters of wireless sensor network. As the sensor nodes are placed randomly in network, sensor node distance has more impact on this time measurement. So it is necessary to study the relationship between the round trip delay time and Sensor node distance. To prove this relationship the other parameters affecting round trip delay time like speed, data transfer rate, number of sensor nodes in RTD path and other request handled by intermediate nodes are either made constant or disabled. A wireless sensor node hardware designed for this purpose consists of microcontroller (ATMEGA 16L) and ZigBee (XBEE S2) wireless communication module. For configuring the ZigBee module of each sensor nodes in round trip delay path X-CTU software is used. After configuring the all sensor nodes the entire network is simulated in real time by using Dock light V1.9 software. The graph plotted between round trip delay time and sensor node distance proves a linear relationship between it.
\end{abstract}

\section{KEYWORDS}

Wireless Sensor Network, Round Trip Delay, Portable Sensor Node.

\section{INTRODUCTION}

The advances in microelectronics and sensor technology made it possible to have of small, low-cost and large number of sensor nodes placed in measuring range through the wireless communication module like ZigBee [8-11]. The purpose is to sense, collect and preprocess the information from all sensor nodes in network. In such wireless sensor network having large number of portable sensor nodes identification of sensor node, locating the distance of target sensor node with respect to reference sensor node[2] and detection of failed sensor node becomes most difficult [3-5]. In case of indoor positing system the sensor node location accuracy needed is in centimeter. Failure detection of sensor node in WSN is essential because failed or malfunctioning sensor node may produce incorrect data or no data, which will affect the overall quality of the entire WSN. Manually checking of such failed sensor node in WSN is troublesome and impossible.

Different techniques or methods like time delay estimation (TDE), Received Signal Strength (RSS) are developed to resolve above issues in WSN [2-4]. The round trip delay and confidence factor is the most powerful tools among them [1].The minimum imposed condition for RTD measurement is that at least 3 sensor node should be present in a loop. This RTD measurement is affected by various parameters of WSN. In WSN having ' $\mathrm{N}$ ' sensor nodes if any one sensor node fails or starts producing 
improper data (malfunctioning) the time delays related to this sensor node will change. This will introduce errors into the RTD time estimates. A brief analysis of RTD time errors can be found in [6]. This paper is arranged into five sections including introduction. Section II gives overview of round trip delay, factors affecting it, limits of sensor nodes in it and round trip delay paths. Section III describes the experimental setup used to prove this relationship. In Section IV real time hardware simulation results are presented. Conclusion is presented in Section V.

\section{ROUND TRIP DELAY}

Round-trip delay (RTD), also called as round-trip time (RTT), is the time required for a signal pulse or packet to travel from a specific source node thru path consisting other nodes and back again. The round trip delay time can range from a few milliseconds (thousandths of a second) under ideal conditions between nearby spaced sensor nodes to several seconds under adverse conditions between sensor nodes separated by a large distance [2]. Let us consider the wireless sensor network having four nodes as shown in figure 1.Consider the minimum condition of having 3 sensor nodes in RTD path. Then in this WSN the maximum RTD paths present will be 4.These are as follows
1. RTD-1 (S1-S2-S3-S1)
2. RTD-2 (S1-S2-S4-S1)
3. RTD-3 (S1-S3-S4-S1)
4. RTD-4 (S2-S3-S4-S2)

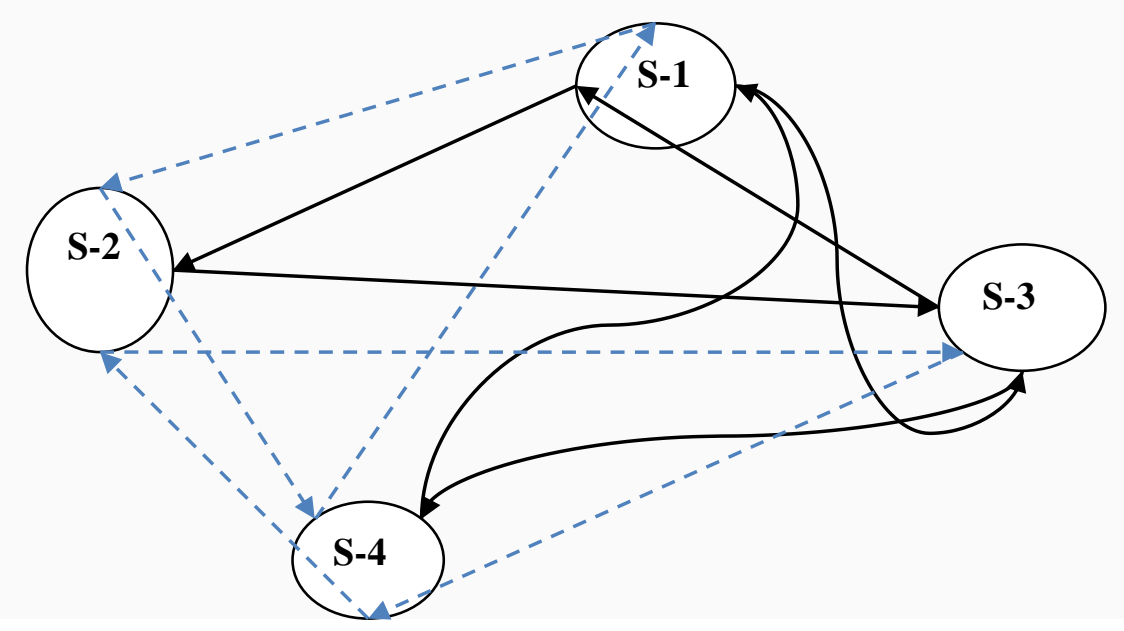

Fig 1: WSN consisting four sensor nodes with maximum four paths for RTD measurement.

The RTD time for RTD-1 path in above network is calculated by using following equation

$$
\mathrm{T}_{\mathrm{RTD}-1}=\tau(1,2)+\tau(2,3)+\tau(3,1)
$$

where $\tau(i, j)$ is the delay time between the sensor node $i$ and $j$ respectively. With the help of this equation-1, the generalized equation for RTD time for the RTD path containing (N-1) sensor node will be written as follows

$$
\mathrm{T}_{\mathrm{RTD}}=\tau(1,2)+\tau(2,3)+------------------+\tau((\mathrm{N}-1), 1)
$$

The round trip delay (RTD) times, with minimum 3 sensor nodes in RTD paths, of all possible paths/orientations in WSN with ' $\mathrm{N}$ ' sensor nodes are listed below in figure 2. 


$$
\left[\begin{array}{c}
\boldsymbol{\tau}_{\mathbf{R T D}-\mathbf{1}} \\
\boldsymbol{\tau}_{\mathbf{R T D}-2} \\
\boldsymbol{\tau}_{\mathbf{R T D}-\mathbf{3}} \\
- \\
- \\
- \\
\boldsymbol{\tau}_{\mathbf{R T D}-(\mathbf{N}-\mathbf{1})}
\end{array}\right]=\left[\begin{array}{c}
\tau(1,2)+\tau(2,3)+\tau(3,1) \\
\tau(1,2)+\tau(2,4)+\tau(4,1) \\
\tau(1,3)+\tau(3,4)+\tau(4,1) \\
- \\
- \\
- \\
\tau(1,2)+\tau(2,(N-1))+\tau((N-1), 1)
\end{array}\right]
$$

Fig 2: RTD paths in WSN with ' $N$ ' sensor nodes

The $\tau(1,2)$ time delay between the sensor node $1 \& 2$ depends upon the distance between them. Time delay is directly proportional to distance and expressed as

$$
\tau(1,2) \propto \mathrm{d}(1,2)
$$

RTD time expressed in equation 1 depends upon the various time delays in the selected path. All these time delays are linear function of distances. Hence RTD time should be a linear function of distance between sensor nodes. Here in this paper it has been proved experimentally.

\subsection{Factors affecting RTD time measurement}

In a network RTD time is affected by several factors. One of them is latency, which is the time between a request for data and the complete return or display of that data. The round trip delay (RTD) time depends on various factors including:

a) Data transfer rate of the sensor node.

b) Nature of the transmission medium.

c) Physical distance between the sensor nodes.

d) Number of nodes in the RTD path.

e) Number of other requests being handled by intermediate nodes.

f) Speed with which intermediate nodes and source node functions.

g) Presence of interference in the circuit.

As stated above the round trip delay time is a function of various parameters of the wireless sensor network and can be expressed by following equation

RTD Time $=\boldsymbol{f}$ (speed, distance, medium, noise, sensor nodes in RTD path \& request handled)

$$
=\mathrm{Ts}+\mathrm{Td}+\mathrm{Tm}+\mathrm{Tn}+\mathrm{Tn}_{\mathrm{RTD}}+\text { Toreq }
$$

A theoretical minimum is imposed on the RTD (minimum 3 sensors in path) because it can never be less than this value. Then it will not form a loop in transmission medium. As the RTD measurement depends upon various parameters $[1,3,5]$ of Sensor Node and WSN. The WSN for various paths round trip delay (RTD) measurements has to be categorized as Symmetrical or Asymmetrical network. A WSN is briefly defined as Symmetrical network if

1) All the sensor nodes are located at equal distance from each other.

2) All sensor nodes should have same sensitivity.

3) Operating speed of all sensor nodes processing unit has to be equal.

4) Same wireless communication module is for all sensor nodes

Otherwise the WSN will be defined as Asymmetrical network.

\subsection{Limitation of sensor nodes in RTD path.}

As mentioned in above point ' $d$ ' round trip delay time depends on the number of sensor nodes present in RTD path. There should be more than 2 sensor nodes to form a round trip in a network. The minimum number of sensor nodes required to form RTD path are 3 [2-4] and maximum sensor nodes in round trip delay paths should not be more than (N-1). This will put the limits on the sensor nodes used in round trip delay path. This limit of sensor nodes in RTD path for WSN with ' $N$ ' sensor nodes is given by

$$
3 \leq \mathrm{m} \leq(\mathrm{N}-1)
$$

where ' $\mathrm{m}$ ' is the number of sensor nodes present in the respective RTD path. 


\subsection{Determination of number of RTD paths in WSN}

The number of RTD paths in WSN depend upon the number of sensor nodes selected for RTD path. Maximum possible RTD paths in WSN having ' $N$ ' sensor nodes can be calculated by following equation

$$
\mathrm{n}_{\mathrm{RTD}}=\mathrm{N}(\mathrm{N}-\mathrm{m})
$$

where ' $\mathrm{n}_{\text {RTD }}$ ' is the number of RTD paths having ' $\mathrm{m}$ ' sensor nodes in WSN consisting ' $\mathrm{N}$ ' sensor nodes.

In the network having less number of sensor nodes, minimum criteria $(\mathrm{m}=3)$ for number of sensor nodes in RTD path can be used. This will results in optimum number RTD paths. But if the number of sensor nodes in WSN are large then minimum condition $(m=3)$ for selection of sensor node should not be used because it will result in large numbers of RTD paths.

\section{EXPERIMENTAL SETUP}

The four microcontroller-based sensor nodes (Slave) and a master (computer) are used here for experimental purpose. Each sensor node consists of ATMEGA 16L microcontroller and XBEE S2 (Digi key) ZigBee pro wireless communication module as shown in figure 3.

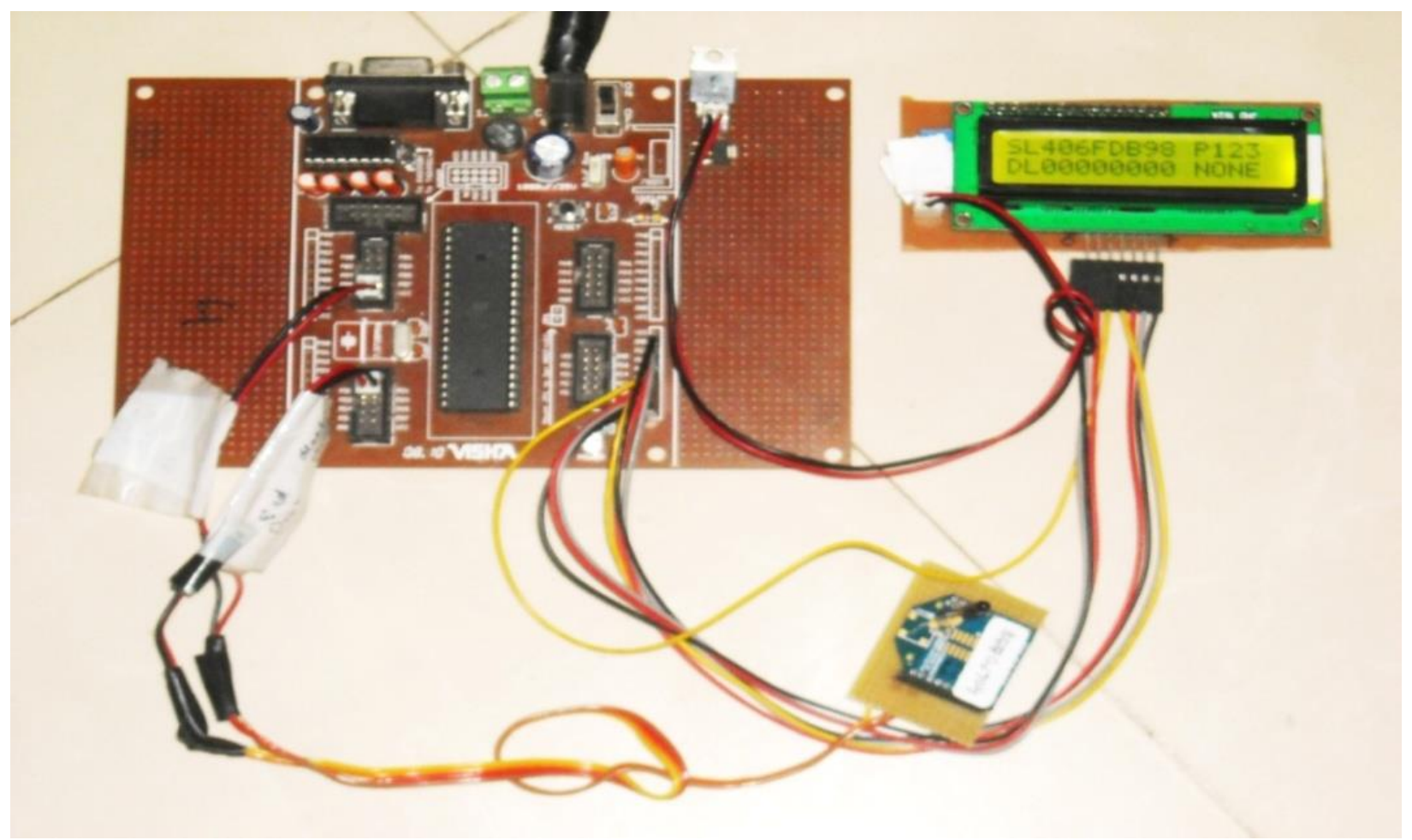

Fig 3: Microcontroller based Sensor node with ZigBee.

\subsection{Hardware Selection and Configuration}

Microcontroller ATMEGA 16L is selected because it is a low-power, high-performance CMOS 8-bit micro-controller with 16K bytes of In-System Programmable Flash memory. The crystal used for microcontroller is $7.3728 \mathrm{MHz}$ with baud rate is set at $9600 \mathrm{bps.LCD}$ display and ZigBee module are interfaced to port $\mathrm{C}$ and $\mathrm{D}$ respectively. The existence of automation device profiles and low power consumption (min.60mW) as compared to Bluetooth makes the ZigBee[9-11] an ideal wireless interface for automation devices [7].The ZigBee addresses of sensor nodes selected in the network have the id as S1 (406FDB22), S2 (406FDBD6) ,S3 (406FD976) and S4(406FDB51).

All ZigBee modules are addressed using Unicast addressing because it supports the retries. As in this application we requires one master and remaining four slaves sensor nodes, ZigBee modules used 
here has to be configured with NonBeacon (w/Coordinator) network technique[8]. In this network configuration technique master sensor node is configured as Coordinator and remaining slave sensor nodes are configured as End Devices. Operation mode selected here is AT Command mode, so that direct transmission will occur between the sensor nodes.

\subsection{Selection of various network conditions}

As the RTD measurement is affected by various parameters of the wireless sensor network mentioned in above equation 4 . The various conditions have to be imposed on the selection of network before applying this method. So except distance other parameters affecting RTD measurement are kept constant. These conditions are mentioned in table 1.

Table 1. Condition Imposed on selected network.

\begin{tabular}{|c|l|l|}
\hline Sr. No. & \multicolumn{1}{|c|}{ Factors Affecting RTD measurement } & \multicolumn{1}{c|}{ Condition Imposed or Chosen } \\
\hline 1 & The data transfer rate of the sensor node & $\begin{array}{l}\text { Similar data rate is used for all sensor } \\
\text { node }(9600 \mathrm{bps})\end{array}$ \\
\hline 2 & The nature of the transmission medium. & Wireless \\
\hline 3 & The physical distance between the sensor nodes & Variable with Symmetrical network \\
\hline 4 & The number of sensor nodes in the RTD path & Minimum (m=03) \\
\hline 5 & $\begin{array}{l}\text { The number of other requests being handled by } \\
\text { intermediate nodes }\end{array}$ & NIL \\
\hline 6 & $\begin{array}{l}\text { The speed with which intermediate nodes and } \\
\text { source node functions }\end{array}$ & Same due identical hardware (in sec) \\
\hline 7 & The presence of interference in the circuit & No (Laboratory Environment) \\
\hline
\end{tabular}

Data transfer rate and speed of communication of sensor nodes are same as ZigBee wireless communication module is used [10]. The minimum condition of sensor nodes $(=3)$ in RTD path is selected here. If more sensor nodes are used in RTD path it will increase the RTD time [3]. This will not affect the linear relationship between the RTD time and sensor node distance.

Here we have to prove the relationship between the RTD time and Sensor Node Distance. So the distance between the sensor nodes is kept variable under symmetrical network conditions. Round trip delay path is kept fix. Sensor nodes are kept at various distances as 1,2,3,4 and 5 feet respectively and then real time results are taken.

\section{Simulation Results}

Initially the each sensor node in WSN as shown in figure 1 is defined for a selected round trip delay path by configuring them with source and destination addresses (of ZigBee module) by using the XCTU software.RTD path selected here is RTD-1 (S1-S2-S3-S1) and the RTD path distance is 1 feet. Sensor node S1 has source address of master and destination address as 406FDBD6, sensor S2 has source address as 406FDB22 and destination address as 406FD976 and for sensor S3 source address is 406FDBD6 and has destination address as 406FDBD22.

After configuring these slave sensor nodes in WSN it is simulated in real time by using the Dock light V1.9 software. The above procedure is repeated for remaining four causes. For these cases sensor nodes are kept at distances 2 feet, 3 feet, 4 feet and 5 feet respectively. Results of simulation are mentioned in table II.

Table 2. Real Time Simulation Results of RTD Time using Dock light V1.9.

\begin{tabular}{|c|c|c|c|}
\hline $\begin{array}{c}\text { Sensor Node } \\
\text { Distance (feet) }\end{array}$ & $\begin{array}{c}\text { Transmitted Time } \\
\text { Tx in sec }\end{array}$ & $\begin{array}{c}\text { Received Time } \\
\text { Rx in sec }\end{array}$ & $\begin{array}{c}\text { Round Trip Delay Time } \\
=\text { Tx-Rx }\end{array}$ \\
\hline 1 & $28: 19.410$ & $28: 34.033$ & $14.623 \mathrm{sec}$ \\
\hline 2 & $28: 56.027$ & $29: 10.651$ & $14.624 \mathrm{sec}$ \\
\hline 3 & $29: 20.023$ & $29: 34.649$ & $14.626 \mathrm{sec}$ \\
\hline 4 & $29: 41.124$ & $29: 55.751$ & $14.627 \mathrm{sec}$ \\
\hline 5 & $31: 09.350$ & $31: 23.978$ & $14.628 \mathrm{sec}$ \\
\hline
\end{tabular}


The RTD time values measured for five different cases are 14.623, 14.624, 14.626, 14.627 and 14.628 $\mathrm{sec}$ respectively. From the readings it observed that as distances changes in 'feet' the RTD time changes in 'msec'. As the distance between sensors nodes in WSN changes, it will change the time delay between the consecutive sensor nodes, which in terms affect the RTD time measurement [2-4]. The change in RTD time against distance between sensor nodes is verified by considering five cases. The round trip delay time (msec) against sensor node distance (feet) graph is shown in figure 5. It gives us the linear relationship between the distance and round trip delay (RTD) time of sensor nodes $[2]$.

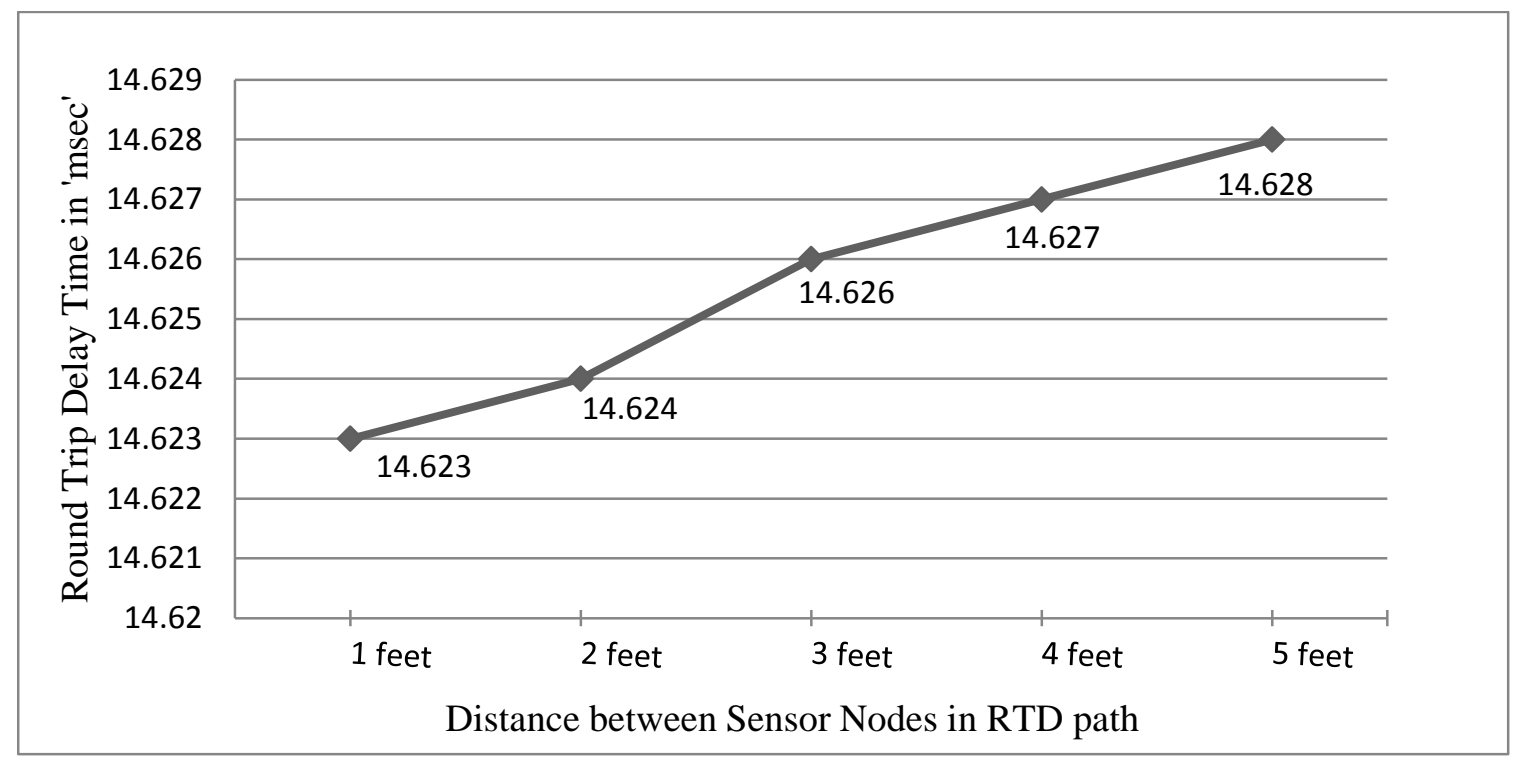

Fig 5: RTD time against Sensor Node Distance.

\section{CONCLUSION}

It is observed from above results that as the distance between the sensor nodes in round trip delay path increases the round trip delay (RTD) time also increases. A In above experimental case the parameters affecting RTD measurement except distance are kept identical. So the round trip delay (RTD) measurement in these cases is purely a function of distance between the Sensor nodes. Hence linear relationship exists between round trip delay time and sensor node distance in WSN.

\section{REFERENCES}

[1] T. W. Pirinen* J. Yli-Hietanen, P. Pertil "a and A. Visa "Detection and compensation of Sensor malfunction in time delay based direction of arrival estimation " IEEE Circuits and Systems, vol.4,May 2004,pp.872-875.

[2] AlessioD Angelis,Antonio Moschitta, Peter Händel and Paolo Carbone "Experimental Radio Indoor Positioning Systems Based on Round-Trip Time Measurement” Advances in Measurement Systems, April 2010,pp.196-219.

[3] J. Yli-Hietanen, K. Koppinen, and J. Astola, "Time-delay selection for robust angle of arrival estimation," in Proceedingsof the IASTED International Conference on Signal and Image Processing, 1999, pp. 81-83.

[4] K. Varma, T. Ikuma, and A. A. Beex, "Robust TDE-based DOA-estimation for compact audio arrays," in Proceedings of the Second IEEE Sensor Array and Multichannel Signal Processing Workshop (SAM 2002), 2002, pp. 214-218.

[5] J. Yli-Hietanen, K. Kallioj"arvi, and J. Astola, "Robust time delay based angle of arrival estimation," in Proceedings of the 1996 IEEE Nordic Signal Processing Symposium (NORSIG96), 1996, pp. 219222.

[6] T. Pirinen, P. Pertil"a, and A. Visa, "Toward intelligent sensors - reliability for time delay based direction of arrival estimates," in Proceedings of the 2003 IEEE International Conference on Acoustics, Speech and Signal Processing (ICASSP'03), 2003, Volume V, pp.197-200. 
[7] Harish R., B. S. Prabhu, Rajit Gadh ,Asad M. Madni "Wireless Industrial Monitoring and Control using a Smart Sensor Platform" accepted for IEEE Sensor Journal 2007.

[8] Corral P., Pena E., Garcia R., Almenar V., de C. Lima, ”Distance Estimation System based on ZigBee "Proceedings of 11th IEEE International Conference on Computational Science and Engineering Workshops, CSEWORKSHOPS '08, San Paulo,16-18 July 2008, pp.405-411,

[9] Cho H., Kang M., Park J., Park B. \& Kim H. "Performance Analysis of Location Estimation Algorithm in ZigBee Networks using Received Signal Strength" Proceeding of 21st IEEE International Conference on Advanced Information Networking and Applications Workshops, AINAW'07,2007,Niagara Falls, CA.

[10] Santinelli G., Giglietti R. \& Moschitta A. "Self-calibrating indoor positioning system based on ZigBee Devices" Proceedings of the IEEE International Instrumentation and Measurement Technology Conference, I2MTC,May 2009, pp. 1205 - 1210.

[11] Schwarzer S., Vossiek M., Pichler M. \& Stelzer A.’Precise distance measurement with IEEE 802.15.4 (ZigBee) devices" IEEE Radio and Wireless Symposium, Orlando, FL, 22-24 Jan. 2008, pp. 779 - 782

\section{Authors}

Ravindra N Duche received the B.Engg. degree in Electronics Engineering from Mumbai University, India, in 1994 and the M.Engg. degree in Electronics Engineering from Shivaji University, India, in 2005. He is currently pursuing his PhD in the field of Smart Sensor.

Nisha Sarwade received the B.E. degree in Electronics Engineering from Jiwaji University, Gwalior in 1975 and the M.E. (Solid state Electronics) and Ph.D. degrees in Electronics engineering from University of Roorkee, in 1979 and 1988 respectively. She worked as Research Associate at University of Roorkee, Roorkee on UGC Project in period 1979-1983. From 1983-1987, she joined, Electronics and Computer Engineering Department, University of Roorkee, Roorkee as Lecturer. In 1988, she joined Veermata Jijabai Technological Institute, Mumbai as lecturer. Since 1996, she has been an Assistant Professor with Veermata

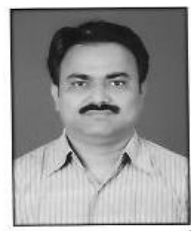
Jijabai Technological Institute, Mumbai. Her research interest includes Carbon nanotubes, Compound semiconductors, High K dielectrics and flash memories. 